\title{
Smart implants in orthopedic surgery, improving patient outcomes: a review
}

This article was published in the following Dove Press journal: Innovation and Entrepreneurship in Health

\author{
Eric H Ledet ${ }^{1,2}$ \\ Benjamin Liddle' \\ Katerina Kradinova' \\ Sara Harper' \\ 'Department of Biomedical \\ Engineering, Rensselaer Polytechnic \\ Institute, Troy, NY, USA; ${ }^{2}$ R\&D Service, \\ Stratton VA Medical Center, Albany, \\ NY, USA
}

\begin{abstract}
Smart implants are implantable devices that provide not only therapeutic benefits but also have diagnostic capabilities. The integration of smart implants into daily clinical practice has the potential for massive cost savings to the health care system. Applications for smart orthopedic implants have been identified for knee arthroplasty, hip arthroplasty, spine fusion, fracture fixation and others. To date, smart orthopedic implants have been used to measure physical parameters from inside the body, including pressure, force, strain, displacement, proximity and temperature. The measurement of physical stimuli is achieved through integration of application-specific technology with the implant. Data from smart implants have led to refinements in implant design, surgical technique and strategies for postoperative care and rehabilitation. In spite of decades of research, with very few exceptions, smart implants have not yet become a part of daily clinical practice. This is largely because integration of current sensor technology necessitates significant modification to the implants. While the technology underlying smart implants has matured significantly over the last several decades, there are still significant technical challenges that need to be overcome before smart implants become part of mainstream health care. Sensors for next-generation smart implants will be small, simple, robust and inexpensive and will necessitate little to no modification to existing implant designs. With rapidly advancing technology, the widespread implementation of smart implants is near. New sensor technology that minimizes modifications to existing implants is the key to enabling smart implants into daily clinical practice.
\end{abstract}

Keywords: smart implant, sensor, strain gage, force, knee, hip, spine, fracture, passive resonator

\section{Introduction}

Smart implants are implantable devices that provide not only therapeutic benefits but also have diagnostic capabilities. Smart implants can enable personalized medicine, optimize care for individual patients and improve outcomes while reducing costs. ${ }^{1}$ As diagnostic tools, smart implants can provide information characterizing the environment inside the body that cannot be obtained by any other way. This information can provide objective quantitative data to tailor treatments, trigger transitions in care and detect adverse events earlier. ${ }^{2,3}$ Smart implants can also provide continuous monitoring of critical intracorporal parameters, the data from which can be used to guide treatments in real time. ${ }^{2}$ The integration of smart implants into daily clinical practice has the potential for massive cost savings to the health care system by minimizing expensive complications, decreasing recovery times, decreasing lost work days after surgery and reducing readmissions and revision procedures. ${ }^{2}$ Smart implant-based research has also contributed critically to our understanding of in vivo pathophysiology,
Correspondence: Eric H Ledet Department of Biomedical Engineering, Rensselaer Polytechnic Institute, I 10 Eighth Street, JEC 7044, Troy, NY 12180 , USA

Tel + I 5182766959

Fax +I 5182763035

Email ledete@rpi.edu
Innovation and Entrepreneurship in Health $20|8: 54|-5 \mid$

41 
healing, implant-tissue interfaces and biomechanics. Smart implant research has also provided important knowledge for the development of next-generation implants and surgical techniques. ${ }^{4,5}$

While the technology underlying smart implants, including sensing, power transfer, energy storage and wireless communications, has matured significantly over the last several decades, there are still significant technical challenges that need to be overcome before smart implants become part of mainstream health care. ${ }^{2,5}$

In all smart implant applications, the implant is the vehicle that carries the diagnostic technology into the body (Figure 1). Owing to the relatively large physical size of many orthopedic implants, the bulk provides an opportunity for symbiosis between implant and sensing technology. ${ }^{6}$ Physically large implants provide the means to incorporate sensors, signal conditioning electronics and telemetry into the implant itself or on its surface. Because of the opportunity for integration of sensing technology, there has been much innovation and development in smart orthopedic implant applications over the last 5 decades.

\section{Technology overview}

Strain gage-based sensing has been the mainstay of smart implants since the $1960 \mathrm{~s} .{ }^{1,4,7}$ Strain gages are thin foil arrays
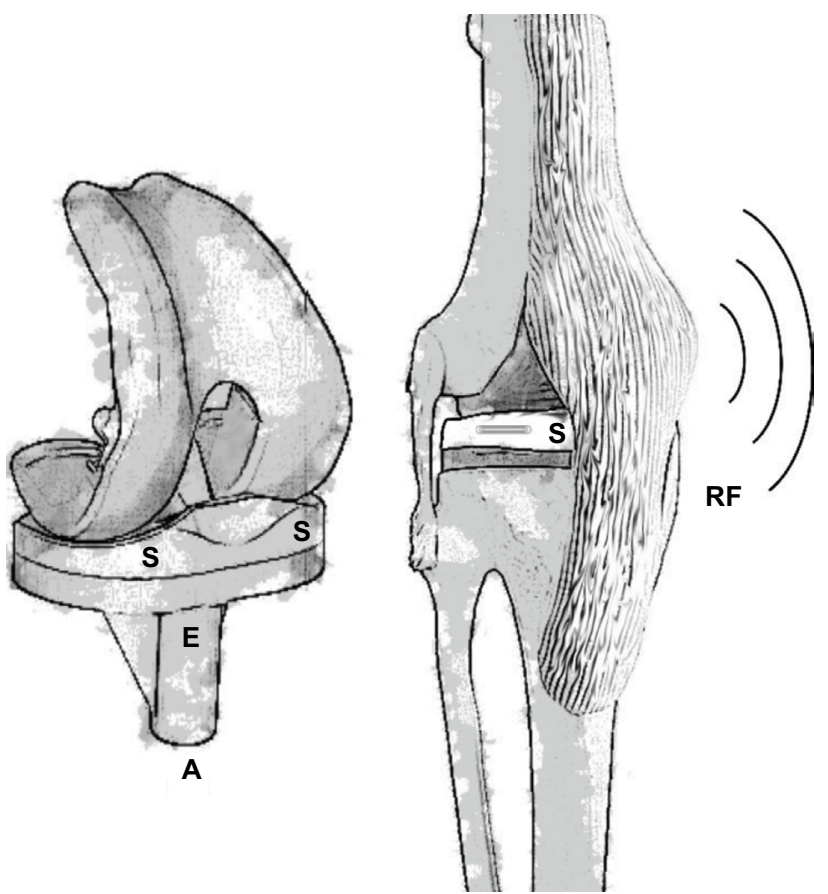

Figure I Orthopedic implants, such as the total knee replacement components shown left, have sufficient size and volume for placement of sensors (S), electronics (E) and antenna (A) components within.

Notes: This facilitates their modification into smart implants. Once placed in the body (right), radiofrequency (RF) communications facilitate data collection from the implant. deposited onto a backing substrate. For smart implant applications, the gage is bonded directly to the surface of the implant with an adhesive (Figure 2). As the implant deforms, the strain gage deforms equivalently, and with that deformation, there is a characteristic change in the resistance of the gage, which is proportional to the strain experienced by the implant. After signal conditioning, the output voltage is proportional to strain. ${ }^{8}$

Protecting strain gages and their circuits from the effects of bodily fluids is a challenge. ${ }^{7,9}$ A strategy to obviate this challenge is to modify the implant in a way where the strain gages can be mounted on the inside of the implant. ${ }^{10,11}$ In many smart implant applications, the implant is modified by creating a cavity or recess into which strain gages can be mounted and the signal conditioning electronics can be placed. ${ }^{1,12}$ Once the sensors and electronics are in the cavity, a lid is laser welded to hermetically seal the cavity. ${ }^{1,11,13}$ The strain gages are sealed within the altered structure of the implant with lead wires or an antenna extending out of the cavity for transfer of power and communication of data. ${ }^{1,14}$

The earliest smart implants utilized percutaneous lead wires that extended from the instrumented implant directly to an external data logger. ${ }^{15,16}$ The obvious limitations of percutaneous lead wires include the potential for infection, limited mobility of the patient and patient acceptability. ${ }^{4}$ While percutaneous connections are not viable for clinical

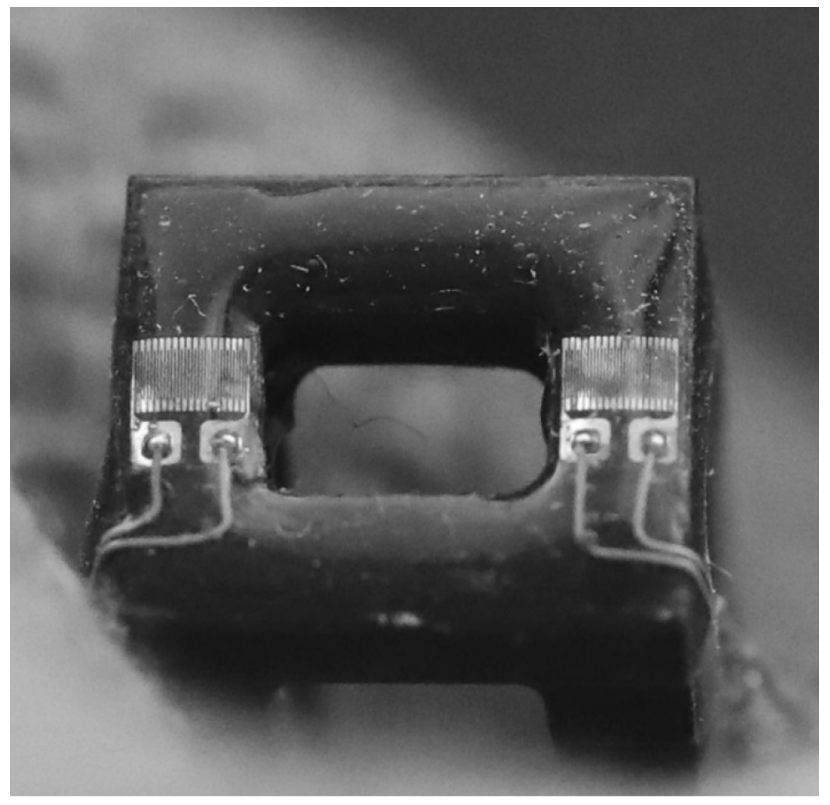

Figure 2 Strain gages are mounted onto the surface of implants such as the cervical spinal interbody cage shown and require lead wires connecting them to signal conditioning electronics.

Note: The gages shown are $2.5 \mathrm{~mm}$ in width and are coated with parylene $\mathrm{C}$ to isolate them from the body. 
applications, ${ }^{1,3}$ wired smart implants do provide a low-cost, high-performance technology for preclinical research. ${ }^{17,18}$

To go beyond the limitations of wired systems, secondgeneration smart implants utilized telemetry transmitters powered by batteries (Figure 3). ${ }^{1,19,20}$ Battery-powered systems provide direct power to the implanted electronics and do not have the disadvantages of percutaneous leads. Battery-powered systems are limited by their large size (to accommodate the bulk of the battery) and the finite life of the battery itself. ${ }^{21}$ Because of their limited life, powering smart implants by batteries is impractical for anything other than acute studies or preclinical research. ${ }^{1,22,23}$

To facilitate a (theoretically) infinite life, wireless, inductively powered smart implant systems have been developed. ${ }^{24}$ These systems rely on the transfer of electromagnetic energy between a source outside the patient and a receiver integrated into the implant. Electromagnetic energy is transmitted by inductive coupling through radiofrequency (RF) fields. The implanted systems have no battery but typically contain energy storage elements that power the circuits once power is inductively transferred.

The earliest inductively powered smart implant systems were developed in the 1960s and 1970s. ${ }^{25-27}$ These systems were generally complex and bulky primarily due to the size of the electrical components available at that time. Multiple printed circuit boards were necessary for interfacing sensors with signal conditioning electronics and transmitting data. ${ }^{25}$ Owing to their complexity, these systems had low reliability

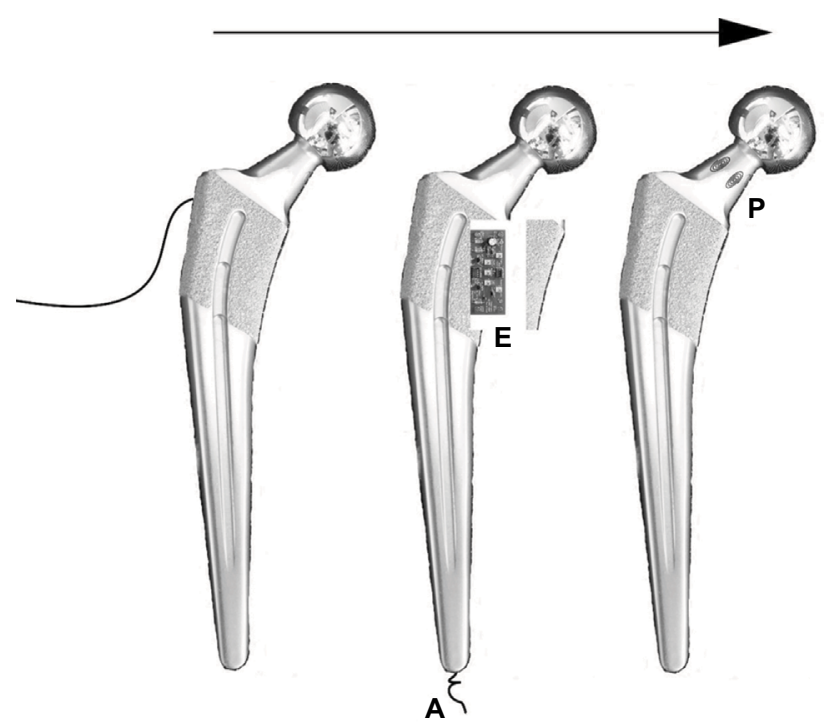

Figure 3 The technology that enables smart orthopedic implants has evolved over several decades, as shown in the total hip prostheses, from tethered electronics (left) to wireless systems that require signal conditioning electronics $(E)$ with antennas $(A)$ to be housed inside the implant (center) to passive sensors $(P)$ that require no electronics and little-to-no modification of the implant (right). and were fraught with failures. As electronics technology evolved, telemetry systems became more compact and more robust, which has enabled smart implant technology to become more viable for clinical applications over the last 2 decades. ${ }^{12}$ Low power circuits have been developed to minimize the amount of power transferred.$^{11}$ These systems can read multiple sensors and have little drift over time., ${ }^{9,10}$

Within the implant, typical smart implants include strain gages, a power coil for inductive coupling, an antenna for transmitting data, signal conditioning circuits and a telemetry system. ${ }^{10}$ External readers generate an RF signal that is transmitted through an external antenna to the implanted system. Individual implants are precalibrated by applying a known stimulus (eg, force or pressure) to the implant in the controlled setting of the laboratory. ${ }^{1,13}$ The corresponding strain signals are used to calibrate the implant. Each implant's calibration data are used to convert the transmitted strain to a signal representative of the measurand.

Smart orthopedic implants have primarily been used to measure physical parameters, including pressure, force, strain, displacement, proximity and temperature. The measurement of physical stimuli is achieved through integration of application-specific technology with the implant. Applications for smart orthopedic implants have been identified for knee arthroplasty, hip arthroplasty, spine fusion, fracture fixation and others.

\section{Applications in the knee}

Osteoarthritis of the knee is one of the most common musculoskeletal pathologies worldwide. For patients who fail conservative therapy, total knee arthroplasty (TKA) is the gold standard treatment. By 2030, the demand for primary total knee arthroplasties is projected to grow to 3.48 million procedures in the USA. ${ }^{28}$ During TKA, the distal femur and proximal tibia are resected and often the patella is resurfaced. The distal femur and proximal tibia are replaced with metal components, and an ultra-high molecular weight polyethylene (UHMWPE) insert is affixed to the tibial component, on which the femoral component articulates. An UHMWPE button is cemented to the posterior patella for articulation of the patellofemoral joint.

Although TKA is a common procedure with low complication rates, postoperative joint biomechanics can affect range of motion, implant survival rates and long-term outcomes. These factors are largely dependent on the surgical technique and implant design. Feedback that indicates forces, pressures, displacements or stresses on the implant intraoperatively and postoperatively can be used to optimize 
implant design, implant selection and surgical technique, all of which affect patient outcomes. ${ }^{29}$ In this way, smart knee implants have an important role in the understanding of knee biomechanics.

Knee forces are highly dynamic and dependent on body weight, external loads, muscle activity and joint kinematics. The magnitude of these forces dictates implant wear rates and implant component survival. Estimating forces across the tibiofemoral and patellofemoral joints remains a challenge due to the complex and redundant contributions of the muscles. Smart implants have been used to measure tibiofemoral joint forces for patients undergoing TKA.

First-generation implants integrated strain gage-based load cells into the tibial tray. ${ }^{30}$ The stem and keel of these components were hollowed out to house signal conditioners, microprocessors and telemetry. ${ }^{31}$ Second-generation designs were capable of measuring multiaxial forces via six or 12 strain gages affixed to the stem distal to the tibial tray. ${ }^{32,33}$

Based on data from smart knee implants, peak forces while walking after TKA are 1.8-2.6 times body weight (BW) and are centered in the middle of the tibial tray. ${ }^{34-37}$ Walking on a treadmill reduces knee forces relative to a hard floor, while increasing the speed of gait increases forces. ${ }^{35}$ Jogging results in forces of $\sim 4 \times \mathrm{BW}$. Forces in the knee are $19 \%$ higher during gait with shoes than without shoes. ${ }^{34}$ During stair climbing, forces increase to $\sim 3.0 \times \mathrm{BW}$ at $35^{\circ}$ to $50^{\circ}$ of knee flexion. ${ }^{37,38}$ Stair decent causes highest forces of $4.0 \times \mathrm{BW}^{36}$ Rising from a chair results in peak forces of $1.5-2.5 \times$ BW. Stationary cycling results in forces of $\sim 1.0 \times$ $\mathrm{BW}$, whereas forces of $4.0 \times \mathrm{BW}$ are generated during golf swing. ${ }^{35}$ Peak forces during tennis are associated with the serve and are lower than golf. ${ }^{35}$ Forces across the knee can exceed $5.5 \times \mathrm{BW}$ when muscles are co-contracted during loss of balance. ${ }^{36}$ During all activities, shear forces are small compared to axial loads. ${ }^{36,39}$

While there has been substantial research using smart implants to better characterize tibiofemoral forces in the knee, little research has been conducted for the patellofemoral joint. The patellofemoral joint is physically small, and thus, there is little room for the placement of sensors, signal conditioning electronics and telemetry within the patellar implant. This has made the development of smart patellar implants prohibitive with traditional technology (such as strain gages). Only recently a smart patellar implant has been developed to measure patellofemoral forces. ${ }^{40}$ Three passive resonator force sensors were integrated with an off-the-shelf UHMWPE patellar button with no modification to the implant in a configuration where all forces transmitted through the patellofemoral joint were also transmitted through the sensors. While this technology has yet to be used in vivo, the simple integration of the sensors with the implant makes this technology promising.

To date, all in vivo applications for permanent smart knee implants have been research oriented and not part of clinical practice. Data from smart implants have led to refinements in implant design, surgical technique and strategies for postoperative care and rehabilitation. Future applications for permanent smart knee implants include monitoring forces during activities to avoid exposing the knee to forces that could accelerate wear, cause loosening or premature failure of the implant.

While in vivo data collection from permanent implants remains challenging, there is substantial clinical value to providing intraoperative measurements of forces using trial implants during TKA. Alignment and sizing of the components intraoperatively are critical to achieve balance and the appropriate mechanical axis of the knee. Collateral ligament release is commonly used to adjust tension between medial and lateral compartments. The extent of release dictates the forces in the two compartments and historically was based on clinical judgment. Smart implants that provide force measurements in the two compartments have been used intraoperatively to guide ligament balance.

For intraoperative measurements, first-generation provisional or trial components were instrumented with four piezoelectric elements to measure forces in the anterolateral, posterolateral, anteromedial and posteromedial quadrants of the knee. ${ }^{41}$ This concept has evolved into a family of smart tibial trial components that are commercialized by OrthoSensor. ${ }^{42}$ The system comprises an array of sensors and a microprocessor, which wirelessly transmits real-time data to a portable graphic display unit that shows forces and points of contact in the component intraoperatively. ${ }^{43}$ OrthoSensor has aligned itself with multiple implant manufacturers to provide smart components that be can used with off-theshelf implants by surgeons who want the additional data intraoperatively. Costs for the instrumented trial implant are $\sim \$ 500$, but this expense is justified in the better outcomes and fewer complications that are fostered with the advanced technology. ${ }^{42-45}$ Other systems with similar designs have recently been described. ${ }^{46}$

\section{Applications in the hip}

Like the knee, osteoarthritis is common in the hip. For patients who fail conservative care, total hip arthroplasty (THA) is the gold standard treatment. By 2030, the demand 
for primary total hip arthroplasties is estimated to grow to $572,000 .{ }^{28}$ During THA, the proximal femur is resected and the acetabulum is resurfaced. The proximal femur is replaced with a metal stem and metal or ceramic ball. A metal cup is placed in the acetabulum with a UHMWPE or ceramic insert on which the femoral component articulates.

Like the knee, biomechanics plays a critical role in implant survival and patient satisfaction following THA. Smart implants have played a significant role in our understanding of the biomechanics of THA and optimizing outcomes.

The first smart THA was performed in $1966 .{ }^{15}$ In this landmark study, a custom three-part femoral component was designed and fabricated with strain gages in the neck. The prosthesis was wired, and the lead wires extended percutaneously from the implant to an external data logger. It was not until almost a decade later that the next-generation wireless prosthesis was developed and used clinically. ${ }^{25}$ These systems incorporated up to 14 strain gages, signal conditioning circuits and battery-powered ${ }^{20}$ or inductively powered $^{47}$ telemetry systems. The sensors and up to five printed circuit boards, which comprised the telemetry and signal conditioning electronics, were placed in the hollow ball $^{25}$ or the hollowed neck ${ }^{20}$ of the femoral component. The longevity of these implants was limited, and initial data were collected for 31 days or fewer. Similar implants were used to measure contact pressure in the hip for up to 36 months postoperatively. ${ }^{48}$

More recent implants measure loads and bending moments in all six directions with $<1 \%$ error. ${ }^{49}$ Some systems have been instrumented with two telemetry systems and with sensors to measure both force and temperature throughout the femoral component. ${ }^{50}$ State-of-the-art implants measure force and temperature with all the electronics contained within the titanium stem (no antenna outside the prosthesis). ${ }^{51}$

Data from smart hip implants indicate that forces are as high as to $3.6 \times \mathrm{BW}$ during one-leg standing, $3.3 \times \mathrm{BW}$ during walking and $4.3 \times \mathrm{BW}$ during running. ${ }^{20,47}$ Data indicate that hip forces during double-leg stance are $\sim 1.0 \times \mathrm{BW}^{20}{ }^{20}$ During stair ascent, forces are up to $2.6 \times \mathrm{BW}^{20,47,52,53}$ Isometric contraction of the hip adductors results in a peak pressure of $5.0 \mathrm{MPa},{ }^{48}$ while pressure as high as $18 \mathrm{MPa}$ was measured during dynamic activities, including rising from a chair, stair climbing, jogging and jumping. ${ }^{48}$ Stationary cycling generates a low maximum pressure of $1.6 \mathrm{MPa}$ and forces of $\sim 1.2 \times \mathrm{BW}^{48,54}$ The highest observed forces occurred during jogging at 12 months postoperatively $(5.6 \times \mathrm{BW})$ and during stumbling $(7.2-8.7 \times \mathrm{BW}),{ }^{14,52}$ suggesting the significant contribution of muscle contraction to joint forces. The choice of footwear (hard or soft heel) does not affect forces during walking. The use of crutches reduces hip forces by $\sim 20 \%$, but only during the first 4 weeks postoperatively, after which the differences diminish. ${ }^{55}$ Forces on the hip are reduced more by using a cane in the contralateral hand than in the ipsilateral hand. ${ }^{15}$

Data also indicate that during walking, temperatures in the hip can exceed $43^{\circ} \mathrm{C}$ in joints with ceramic ball and UHMWPE cup. ${ }^{56}$ Temperatures were lower in joints with alumina ceramic cups.

In addition to force, pressure and temperature measurements, smart hip implants have also been developed to detect loosening of prostheses, which is one of the most common complications of THA. ${ }^{57}$ Implants were instrumented with vibration-sensitive lock-in amplifiers and telemetry. When the femur was subjected to vibrations during simulations, the systems were able to detect loosening.

Like the knee, the motivation for the in vivo smart hip implant use to date has been research applications, not for guiding patient-specific care. Data gleaned from smart hip implants have addressed many clinically relevant questions to direct care. Smart implants have provided answers to questions about the use of crutches or canes postoperatively and have guided rehabilitation regimens following THA. ${ }^{48}$ As research tools, data from smart hip implants have helped to better define load conditions for testing and verifying the performance of implants in vitro. ${ }^{58}$ As tools for designing the next generation of implants and materials, they have provided a more comprehensive understanding of the biomechanical demands of THA.

\section{Applications in the spine}

Low back pain and neck pain are the leading causes of disability worldwide..$^{59,60}$ After failing conservative care, many patients elect to undergo spinal fusion surgery. There are $>450,000$ spinal fusions each year in the USA.$^{61}$ The goal of spinal fusion surgery is to perform an arthrodesis between two (or more) adjacent vertebrae and facilitate bony bridging between the vertebrae. In the cervical spine, this is typically achieved by placing an interbody "cage" implant into the intervertebral disk space and then affixing an anterior cervical plate to the adjacent vertebral bodies. In the lumbar spine, there are multiple surgical options, among the most common being posterior decompression and fusion. A laminectomy is performed to decompress the spine, an interbody cage is placed in the intervertebral disc space and pedicle screws and rods are used to stabilize the spine posteriorly. 
The success or failure of a spinal fusion is highly dependent on both biology and biomechanics. Yet the biomechanics of the spine is poorly understood. Loading is extremely complex due to the multiply redundant intrinsic and extrinsic muscles acting on the spine. After surgery, implants are subjected to axial forces as well as bending moments in flexion, extension, lateral bending and torsion. An understanding of these forces is critical to selecting the appropriate intervention, designing effective implants and prescribing optimal postoperative rehabilitation.

Smart implants have been used as research tools to understand spine biomechanics since 1966 when Waugh ${ }^{16}$ instrumented Harrington rods with 10 strain gages and implanted them into three patients undergoing fusion for scoliosis. The instrumented rods were placed in the spine temporarily and were used to collect force data until they were exchanged for traditional rods in a follow-up procedure. The smart rods had lead wires, which extended percutaneously and attached directly to a data logger. Later, a modified version of smart Harrington rods was developed to have the rods function as variable inductance transducers whereby the inductance changed with application of force. The transducer was attached to a telemetry system, and the smart rods were placed in four patients. ${ }^{26,62}$ To overcome some of the limitations of these first-generation rods, next-generation systems were enabled by mounting strain gages to the hooks that attached the rod to the spine. ${ }^{63}$ The strain gages were attached to lead wires, which extended percutaneously. The systems were used intraoperatively to measure forces during spinal distraction in the correction of scoliosis.

As the use of Harrington rods decreased, next-generation smart spinal implants were developed using similar strategies as total knee and hip components; hollow spaces were created in large fixators to house strain gages, signal conditioning electronics and telemetry systems. ${ }^{64,65}$ The only spine fixators physically large enough were rods placed on the posterior spine in a configuration where they were loaded in parallel (ie, load sharing) with the spine. The implants were instrumented to measure forces and bending moments in all directions. ${ }^{66}$

Data from these posterior fixators demonstrated forces as high as $363 \mathrm{~N}$ during coughing and $676 \mathrm{~N}$ when one patient vomited. ${ }^{26}$ The high forces were likely due to muscle contration. ${ }^{67}$ Forces during sitting have been measured at $176 \mathrm{~N},{ }^{16}$ and forces during twisting and lifting (a $2 \mathrm{~kg}$ weight) can exceed $421 \mathrm{~N} .{ }^{26}$ Peak bending moments on the rods were as high as $9.0 \mathrm{Nm}$, and peak compression forces approached $400 \mathrm{~N}$ during flexion. ${ }^{67,68}$ The application of a cast or a Milwaukee brace reduced forces on the rods, ${ }^{62}$ and the use of a walker reduced forces in the spine by $\sim 25 \%{ }^{68}$ Ascending and descending stairs resulted in forces approximately equal to level walking. ${ }^{68}$

As research tools, forces measured by posterior rods have provided valuable insights into the biomechanical environments to which the rods are exposed, but because the rods are load sharing with the spine, forces in the spine itself cannot be determined in this way. In contrast to posterior rod-based systems, interbody implants and corpectomy implants (vertebral body replacements) are loaded in series with the spine, and thus, they are exposed to the same forces to which the spine is exposed. Like hip and knee implants, vertebral body replacements are sufficiently large to house strain gages, signal conditioning electronics and telemetry within..$^{69}$ However, interbody implants are much smaller than smart hip, knee and posterior spinal implants, and so different strategies have been used for these systems. For interbody cages, strain gages have been used to transduce force, but due to their small physical size, these systems have either required lead wires that extend percutaneously ${ }^{17,18}$ or the implants were connected to telemetry systems that were placed outside of the spine in a subcutaneous pouch..$^{22,23}$

Data from preclinical models indicate that forces on the spine are highly dynamic, dependent on activity, posture and muscle contraction, and can exceed $4.7 \times \mathrm{BW}^{22,23}$ Highest forces were achieved during combined flexion with torsion, and the magnitude of forces correlated strongly to muscle contraction. ${ }^{17}$ Recently, instrumented interbody implants have demonstrated that the mechanical properties of the implants themselves dictate load sharing, which can strongly affect the rate and quality of fusion. ${ }^{70}$

Clinical data from smart interbody implants and vertebral body replacements show that forces are highly dynamic. From sitting or standing in neutral position, flexing even a few degrees causes a significant increase in compressive forces. During flexion, forces in the spine can exceed $5 \times \mathrm{BW}^{71}$ Carrying loads in the arms causes axial forces in the spine to exceed $700 \mathrm{~N} .{ }^{71}$ Lifting a $10 \mathrm{~kg}$ weight from the ground results in forces exceeding $1,650 \mathrm{~N} .{ }^{72}$ When walking, the use of a walker reduces spinal forces by up to $60 \% .{ }^{71}$ When lying down, forces are reduced to $<100 \mathrm{~N}$ whether supine, prone, or lateral. ${ }^{11}$ Highest moments occur during lateral bending and can exceeded $5 \mathrm{Nm}$ in magnitude. ${ }^{71,73}$

One of the most significant clinical challenges following spine fusion surgery is determining when solid bony fusion has occurred. Smart spinal implants may be a means to quantitatively assess the progression of fusion because 
forces through implants change as bone is forming and fusion is maturing. ${ }^{74-77}$ In an attempt to achieve this important clinical goal, new techniques have been proposed for measuring forces to detect fusion in pedicle screw-based smart implants ${ }^{74,75,78}$ and interbody implants. ${ }^{79}$ Although, no correlations have been found between time since surgery and forces measured in smart implants to date ${ }^{80,81}$ just recently, promising new research with interbody cages has shown that forces on smart interbody implants do correlate to the progression of fusion. ${ }^{82}$

In the spine, smart implants have provided critical data on the role of posture, activity and muscle activation in spine biomechanics. This is important because spine loading plays a key role in both the disease process and the course of healing for patients with low back pain who undergo surgical intervention. These data have directed researchers and clinicians on optimizing research models, refining implant designs and managing postoperative care and rehabilitation. Only recently, however, have smart spine implants shown promise as tools to objectively and quantitatively diagnose the progression of fusion. This has the potential to guide care and optimize treatments for individual patients. However, this clinical application has not yet been realized.

\section{Applications in fracture fixation}

In surgical fixation of long bone fractures, an implant is affixed to the bone both proximal and distal to the fracture to act as a buttress. The implants stabilize the bony fragments to facilitate healing. Fracture plates, intramedullary rods and external fixators are options for fracture fixation. When a bone is loaded (eg, the tibia is loaded during weight bearing on the lower limb), the loads are transmitted through the bone and the fixator (Figure 4). In the acute postoperative period, the fracture is not capable of sustaining any loads, and thus if the patient bears weight on the limb, the forces are transmitted exclusively through the fixator and not the bone. As the fracture consolidates and a bony callus forms, the bone is able to bear some load and thus the fixator experiences less force. As the fracture heals and bone bridges, the fracture is able to bear more load while less force is transmitted through the fixator. In this way, monitoring loads on a smart fracture fixation device during weight bearing can be used as an indication of consolidation and healing of a fracture. ${ }^{83-87}$

Forces measured from smart fracture fixation devices can be used to provide objective data to guide rehabilitation strategies for different stages of treatment (eg, to determine when weight bearing can be allowed, ${ }^{85,87}$ to determine objectively if a patient is progressing toward a nonunion, ${ }^{84,88}$ to guide patients

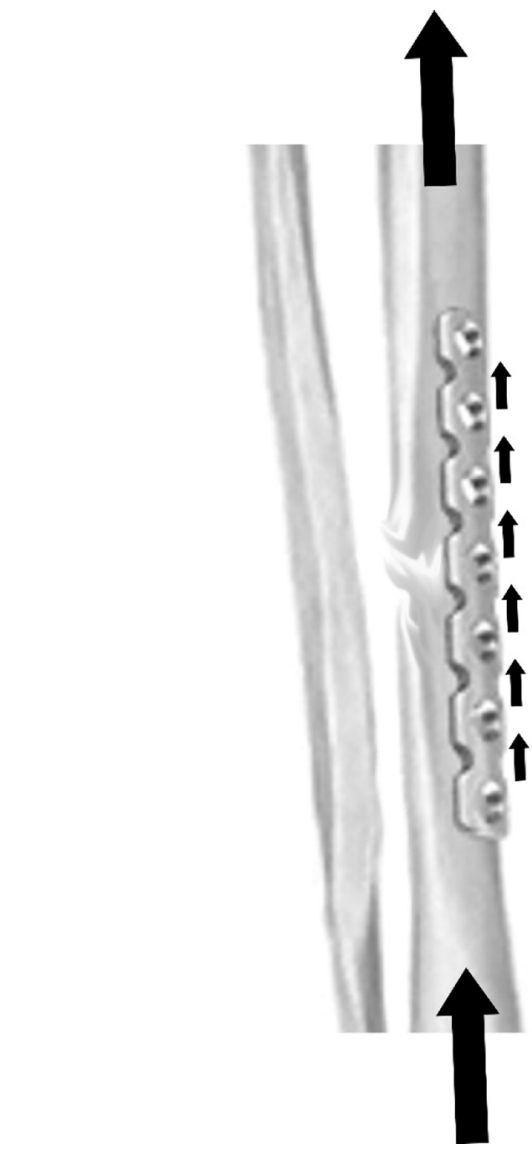

Figure 4 When a fracture is treated with open reduction and internal fixation, forces applied through the bone are transmitted through the plate.

Notes: As shown in the tibia, initially, the fracture is not capable of bearing forces. However, as the fracture heals, the newly formed bone can bear more load, while the plate shares less load. In this way, monitoring forces through the plate is used as an indicator of fracture healing.

on the most effective weight-bearing exercises to stimulate bone formation ${ }^{89}$ and to diagnose when a patient is sufficiently healed to return to work or activities of daily living).

Smart fracture fixation devices have been utilized to better understand the biomechanics of fracture healing for $>40$ years. ${ }^{83,84,90}$ The most comprehensive data are from strain gage instrumented external fixators with direct lead wire connections. ${ }^{1,83,84,90}$ Femoral nail plates have also been instrumented with strain gages and battery-powered telemetry to measure bending moments in the hip. ${ }^{19}$ Large endoprostheses for the proximal femur ${ }^{89,91}$ and distal femur ${ }^{92}$ have been used to house strain gages, electronics and telemetry systems to measure force. Fracture plates have been instrumented with strain gages to measure forces, ${ }^{93,94}$ and intramedullary rods have been hollowed to house strain gages and telemetry for force measurement. ${ }^{86,95}$

Data demonstrate that muscle forces contribute substantially to loading across fractures. ${ }^{89,92}$ Forces in the proximal 
femur (as high as $4 \times \mathrm{BW}$ ) correlate to vastus lateralis and erector spinae activation. ${ }^{89}$ Bending moments in the proximal femur can exceed $20 \mathrm{Nm}$ during activities such as walking and raising the pelvis from a supine position. ${ }^{19}$ Implant forces approach 3,000 $\mathrm{N}$ during walking and jumping. ${ }^{89} \mathrm{In}$ a femoral intramedullary rod, forces exceed $300 \mathrm{~N}$ during partial weight-bearing exercises. ${ }^{86}$ In the distal femur, jogging results in loads of more than $3.3 \times \mathrm{BW}^{92}$

Clinically, force data from smart implants have been used to monitoring loads on fracture plates to ensure that the plate will not fail mechanically. ${ }^{1}$ In the acute postoperative period, patients were instructed to reduce weight bearing on their injured limb until the loads in the fracture plate were below the endurance limit of the plate. Based on this patient-specific guidance, there were fewer failures of the implants, which were monitored. ${ }^{1}$

\section{Challenges and emerging technologies}

In spite of decades of research, with very few exceptions, smart implants have not yet become a part of daily clinical practice. This is primarily because there are a number of limitations and challenges that have yet to be overcome in smart implant technology.

For systems with complex electronics, technical challenges include power consumption, communication range, data transfer rates, size, robustness and cost. ${ }^{5,6,96,97}$ To address power consumption challenges, ultra-low power circuits coupled with energy harvesting strategies have been explored. ${ }^{24}$ Using these strategies, energy is generated in the implant from sources such as vibrations, rotations and deformations during activities such as walking. ${ }^{97}$ While energy harvesting strategies show promise, the quantity of energy available for harvest is not sufficient to power the electronics. ${ }^{24}$

To reduce the size of sensors and signal conditioning circuits, microelectromechanical systems (MEMS)-based technology has been used for smart implant applications..$^{98-101}$ The microscale components and well-established fabrication methods make MEMS attractive for custom electronic and sensor applications. ${ }^{100}$ MEMS sensors can be fabricated from biocompatible materials and materials commonly used in orthopedic implants, including polyethylene, titanium and parylene. ${ }^{29,98}$ Smaller sensors require less power but generally function at higher frequencies (hundreds of megahertz to gigahertz). ${ }^{100}$ One additional challenge at higher frequencies is that at higher frequencies, more energy is absorbed by the tissues, which can cause heating and attenuation of the signal between external electronics and implanted sensor. ${ }^{96}$
MEMS-based strain ${ }^{100}$ and pressure ${ }^{98}$ sensors have been developed and tested. While many MEMS-based transducers have been designed for use in orthopedic smart implants, testing has been limited to bench work and preclinical models.

Perhaps, the most significant barrier to integration into clinical practice has been the necessity for host implant modification to accommodate sensors and electronics. ${ }^{5,6}$ Creating hollow cavities to house complex electronics and strain gages (Figure 3) is technically challenging and expensive and, most significantly, alters the properties of the implant and ultimately jeopardizes the implant's performance. ${ }^{6}$ Sensors for next-generation smart implants will be small, simple, robust and inexpensive and will necessitate little to no modification to existing implant designs. ${ }^{4}$

Recently, piezoresistive polymers have been introduced as an enabling technology for smart orthopedic implants. The inherent electrical properties (resistance) of these composite polymers change when loads are applied to it. Thus, a piezoresistive polymer with low wear rates and good biocompatibility could be used as a force-sensing smart implant in applications where UHMWPE is used today, including knee, hip and shoulder arthroplasty. ${ }^{102}$ Similarly, force sensors and their signal conditioning circuits that are integrated into the polyethylene insert have also been developed. ${ }^{101}$

Passive resonators are an alternative to traditional sensor systems because passive resonator sensors do not require power or signal conditioning electronics. ${ }^{5,6,100,103,104}$ Passive resonator sensors are generally small, simple and comprise few components. They do not require signal conditioning or telemetry because when they are exposed to an RF field (via an antenna), they resonate. The frequency at which they resonate indicates the state of the sensor. A sensor's fundamental (resting, unstimulated) frequency is proportional to the electrical characteristics of the sensor (capacitance and inductance). If designed appropriately, when the sensor is subjected to a change in the stimulus of interest (eg, force, pressure, or strain), the stimulus causes a physical change in capacitance or inductance (or both) of the sensor that shifts the resonant frequency of the sensor. The resonant frequency of the sensor can be detected using an external antenna.

Recently, a family of passive resonator-based sensors has been described that are wireless, battery less, telemetry less and require no electrical connections. ${ }^{40,103-105}$ The small, simple sensors are inexpensive to fabricate $(<\$ 1)$ and can be configured in various sizes and shapes to measure parameters including force, pressure, temperature, $\mathrm{pH}$ and specific target analytes. Because of their small size and simplicity, these sensors have the potential to be integrated into off-the-shelf 
implants will little to no modification of the implant (Figure 3). While this technology has only been tested in simulated in vivo environments and in vitro to date, it shows immense promise for future smart implant applications.

\section{Conclusion}

The clinical utility of smart implants has been conclusively demonstrated, and the potential of the technology to affect clinical care and enable personalized medicine is vast. However, to date, the barriers to entry have made the use of smart implants in daily clinical practice prohibitive. In 5 decades of research, the total number of permanent smart orthopedic implants utilized clinically in all applications is $\sim 100$ patients. However, with rapidly advancing technology, the widespread implementation of smart implants is near. New sensor technology that minimizes modifications to existing implants is the key to enabling smart implants into daily clinical practice.

\section{Disclosure}

Eric H Ledet is co-inventor of US patent 9,662,066 and cofounder and equity holder in inSense Medical, LLC. The other authors report no conflicts of interest in this work.

\section{References}

1. Burny F, Donkerwolcke M, Moulart F, et al. Concept, design and fabrication of smart orthopaedic implants. Med Eng Phys. 2000;22(7):469-479.

2. Andreu-Perez J, Leff DR, Ip HM, Yang GZ. From wearable sensors to smart implants-toward pervasive and personalized healthcare. IEEE Trans Biomed Eng. 2015;62(12):2750-2762.

3. O'Connor C, Kiourti A. Wireless sensors for smart orthopedic implants. J Bio Tribo Corros. 2017;3:20.

4. Sell P. Instrumented implants in orthopedics. J Biomed Eng. 1989;11(2):111-112.

5. Ledet EH, D'Lima D, Westerhoff P, Szivek JA, Wachs RA, Bergmann G. Implantable sensor technology: from research to clinical practice. J Am Acad Orthop Surg. 2012;20(6):383-392.

6. Korduba L, Grabowsky M, Uhl RL, Hella MM, Ledet EH. RFID as a testbed for integration of low frequency RF sensors into orthopaedic implants. J Med Devices. 2013;7:011008.

7. Roberts V. Strain gage techniques in biomechanics. Exp Mech. 1966;6(3):19A-22A.

8. Murray WM, Miller WR. The Bonded Electrical Resistance Strain Gage: An Introduction. New York: Oxford University Press; 1992.

9. Graichen F, Rohlmann A, Bender A, et al. Instrumented Orthopaedic Implants for Wireless Load Measurements. Berlin: Technical Aids for Rehabilitation; 2007:85-86.

10. Graichen F, Bergmann G. Four-channel telemetry system for in vivo measurement of hip joint forces. J Biomed Eng. 1991;13(5):370-374.

11. Graichen F, Arnold R, Rohlmann A, Bergmann G. Implantable 9-channel telemetry system for in vivo load measurements with orthopedic implants. IEEE Trans Biomed Eng. 2007;54(2):253-261.

12. Bergmann G, Graichen F, Siraky J, Jendrzynski H, Rohlmann A. Multichannel strain gauge telemetry for orthopaedic implants. J Biomech. 1988;21(2):169-176.
13. Bergmann G, Graichen F, Rohlmann A, et al. Design and calibration of load sensing orthopaedic implants. J Biomech Eng. 2008;130(2): 021009.

14. Bergmann G, Graichen F, Rohlmann A. Hip joint contact forces during stumbling. Langenbecks Arch Surg. 2004;389(1):53-59.

15. Rydell NW. Forces acting on the femoral head-prosthesis. A study on strain gauge supplied prostheses in living persons. Acta Orthop Scand. 1966;37(Suppl 88):1-132.

16. Waugh $\mathrm{T}$. Intravital measurements during instrumental correction of idiopathic scoliosis. Acta Orthop Scand. 1966;93:58-75.

17. Wachs R, Grabowsky M, Glennon JC, et al. In vivo loads in the cervical spine: a preliminary investigation using a force sensing implant. Spine J. 2012;12:S141.

18. Ledet EH, Peterson J, Wachs RA, Grabowsky MBM, Glennon J, DiRisio DJ. Direct measure of cervical interbody forces in vivo: load reversal after plating. Spine J. 2016;16(10):S362-S363.

19. Brown R, Brustein A, Frankel V. Telemetering in vivo loads from nail plate implants. J Biomech. 1982;15(11):815-823.

20. Davy D, Kotzar G, Brown R, et al. Telemetric force measurements across the hip after total arthroplasty. J Bone Joint Surg. 1988;70(1): $45-50$.

21. van Gaalen JB, Trejos AL, Nikolov HN, et al. Versatile smart hip implant technology using 3D metal printing. 2016 IEEE International Symposium on Circuits and Systems (ISCAS). Montreal, QC: IEEE; 2016: 2731-2734.

22. Ledet E, Sachs B, Brunski J, Gatto CE, Donzelli PS. Real-time in vivo loading in the lumbar spine. Part 1. Interbody implant: load cell design and preliminary results. Spine. 2000;25(20):2595-2600.

23. Ledet EH, Tymeson MP, DiRisio DJ, Cohen B, Uhl RL. Direct realtime measurement of in vivo forces in the lumbar spine. Spine $J$. 2005;5(1):85-94.

24. dos Santos MPS, Ferreira JA, Ramos A, et al. Instrumented hip implants: electric supply systems. J Biomech. 2013;46(15):2561-2571.

25. Carlson CE, Mann RW, Harris WH. A radio telemetry device for monitoring cartilage surface pressures in the human hip. IEEE Trans Biomed Eng. 1974:257-264.

26. Nachemson A, Elfstrom G. Intravital wireless telemetry of axial forces in Harrington distraction rods in patients with idiopathic scoliosis. J Bone Joint Surg. 1971;53(3):445-465.

27. Atkinson J, Shurtleff D, Foltz E. Radio telemetry for the measurement of intracranial pressure. J Neurosurg. 1967;27(5):428-432.

28. Kurtz S, Ong K, Lau E, Mowat F, Halpern M. Projections of primary and revision hip and knee arthroplasty in the United States from 2005 to 2030. J Bone Joint Surg Am. 2007;89(4):780-785.

29. Evans BM, Mahfouz MR, Pritchard ER. Biocompatible MEMS electrode array for determination of three-dimensional strain. Engineering in Medicine and Biology Society, 2006. EMBS'06. 28th Annual International Conference of the IEEE. IEEE; 2006:4092-4095.

30. Kaufman KR, Kovacevic N, Irby SE, Colwell CW. Instrumented implant for measuring tibiofemoral forces. J Biomech. 1996;29(5): 667-671.

31. D'Lima DD, Townsend CP, Arms SW, Morris BA, Colwell CW Jr. An implantable telemetry device to measure intra-articular tibial forces. J Biomech. 2005;38(2):299-304.

32. Kirking B, Krevolin J, Townsend C, Colwell CW Jr, D'Lima DD. A multiaxial force-sensing implantable tibial prosthesis. $J$ Biomech. 2006;39(9):1744-1751.

33. Heinlein B, Graichen F, Bender A, Rohlmann A, Bergmann G. Design, calibration and pre-clinical testing of an instrumented tibial tray. J Biomech. 2007;40(Suppl 1):S4-S10.

34. D'Lima DD, Patil S, Steklov N, Slamin JE, Colwell CW Jr. The Chitranjan Ranawat award: in vivo knee forces after total knee arthroplasty. Clin Orthop Relat Res. 2005;440:45-49.

35. D'Lima DD, Steklov N, Patil S, Colwell CW Jr. The Mark Coventry award: in vivo knee forces during recreation and exercise after knee arthroplasty. Clin Orthop Relat Res. 2008;466(11):2605-2611. 
36. Kutzner I, Heinlein B, Graichen F, et al. Loading of the knee joint during activities of daily living measured in vivo in five subjects. J Biomech. 2010;43(11):2164-2173.

37. Heinlein B, Kutzner I, Graichen F, et al. Complete data of total knee replacement loading for level walking and stair climbing measured in vivo with a follow-up of 6-10 months. Clin Biomech. 2009;24(4): 315-326.

38. Mündermann A, Dyrby CO, D'Lima DD, Colwell CW Jr, Andriacchi TP. In vivo knee loading characteristics during activities of daily living as measured by an instrumented total knee replacement. J Orthop Res. 2008;26(9):1167-1172.

39. D'Lima DD, Patil S, Steklov N, Chien S, Colwell CW Jr. In vivo knee moments and shear after total knee arthroplasty. J Biomech. 2007;40(Suppl 1):S11-S17.

40. Dion M, Drazan J, Abdoun K, et al. Smart orthopaedic implants: applications in total knee arthroplasty. Am J Eng Appl Sci. 2016;9: 1232-1238.

41. Almouahed S, Gouriou M, Hamitouche C, Stindel E, Roux C. Design and evaluation of instrumented smart knee implant. IEEE Trans Biomed Eng. 2011;58(4):971-982.

42. Cassak D. OrthoSensor: will sensor-enabled data transform orthopedics? OrthoSensor is trying to bring a data-driven revolution to orthopedics based on a novel premise: that the key to improved outcomes lies in better surgical technique, not better implants. In Vivo Bus Med Rep. 2011;29:28.

43. Gustke KA, Golladay GJ, Roche MW, Jerry GJ, Elson LC, Anderson CR. Increased satisfaction after total knee replacement using sensorguided technology. Bone Joint J. 2014;96-B(10):1333-1338.

44. Gustke KA, Golladay GJ, Roche MW, Elson LC, Anderson CR. A new method for defining balance: promising short-term clinical outcomes of sensor-guided TKA. J Arthroplasty. 2014;29(5):955-960.

45. Geller JA, Lakra A, Murtaugh T. The use of electronic sensor device to augment ligament balancing leads to a lower rate of arthrofibrosis after total knee arthroplasty. J Arthroplasty. 2017;32(5): 1502-1504.

46. Roth JD, Howell SM, Hull ML. An improved tibial force sensor to compute contact forces and contact locations in vitro after total knee arthroplasty. J Biomech Eng. 2017;139(4):1-8.

47. English T, Kilvington M. In vivo records of hip loads using a femoral implant with telemetric output. J Biomed Eng. 1979;1(2):111-115.

48. Hodge WA, Carlson KL, Fijan RS, et al. Contact pressures from an instrumented hip endoprosthesis. J Bone Joint Surg Am. 1989;71(9):1378-1386.

49. Damm P, Graichen F, Rohlmann A, Bender A, Bergmann G. Total hip joint prosthesis for in vivo measurement of forces and moments. Med Eng Phys. 2010;32(1):95-100.

50. Graichen F, Bergmann G, Rohlmann A. Hip endoprosthesis for in vivo measurement of joint force and temperature. J Biomech. 1999;32(10):1113-1117.

51. Bergmann G, Graichen F, Dymke J, Rohlmann A, Duda GN, Damm P. High-tech hip implant for wireless temperature measurements in vivo. PLoS One. 2012;7(8):e43489.

52. Bergmann G, Graichen F, Rohlmann A. Hip joint loading during walking and running, measured in two patients. J Biomech. 1993;26(8):969-990.

53. Bergmann G, Deuretzbacher G, Heller M, et al. Hip contact forces and gait patterns from routine activities. J Biomech. 2001;34(7):859-871.

54. Damm P, Dymke J, Bender A, Duda G, Bergmann G. In vivo hip joint loads and pedal forces during ergometer cycling. J Biomech. 2017;60:197-202.

55. Damm P, Schwachmeyer V, Dymke J, Bender A, Bergmann G. In vivo hip joint loads during three methods of walking with forearm crutches. Clin Biomech (Bristol, Avon). 2013;28(5):530-535.

56. Bergmann G, Graichen F, Rohlmann A, Verdonschot N, van Lenthe GH. Frictional heating of total hip implants. Part 1: measurements in patients. J Biomech. 2001;34(4):421-428.
57. Marschner U, Grätz H, Jettkant B, et al. Integration of a wireless lockin measurement of hip prosthesis vibrations for loosening detection. Sens Actuators A Phys. 2009;156(1):145-154.

58. Bergmann G, Graichen F, Rohlmann A, et al. Realistic loads for testing hip implants. Biomed Mater Eng. 2010;20(2):65-75.

59. Murray CJ, Vos T, Lozano R, et al. Disability-adjusted life years (DALYs) for 291 diseases and injuries in 21 regions, 1990-2010: a systematic analysis for the Global Burden of Disease Study 2010. Lancet. 2012;380(9859):2197-2223.

60. Murray C, Lopez A. Measuring the global burden of disease. N Engl J Med. 2013;369(5):448-457.

61. Lee J. Rethinking spine care. Mod Healthc. 2014;44(12):14-16.

62. Elfstrom G, Nachemson A. Telemetry recordings of forces in the Harrington distraction rod: a method for increasing safety in the operative treatment of scoliosis patients. Clin Orthop Relat Res. 1973;93:158-172.

63. Daniels AU, Gemperline P, Grahn AR, Dunn HK. A new method for continuous intraoperative measurement of Harrington rod loading patterns. Ann Biomed Eng. 1984;12(3):233-246.

64. Rohlmann A, Bergmann G, Graichen F. A spinal fixation device for in vivo load measurement. J Biomech. 1994;27(7):961-967.

65. Graichen F, Bergmann G, Rohlmann A. Patient monitoring system for load measurement with spinal fixation devices. Med Eng Phys. 1996;18(2):167-174.

66. Rohlmann A, Bergmann G, Graichen F, Mayer HM. Telemetrized load measurement using instrumented spinal internal fixators in a patient with degenerative instability. Spine. 1995;20(24):2683-2689.

67. Rohlmann A, Bergmann G, Graichen F, Mayer HM. Influence of muscle forces on loads in internal spinal fixation devices. Spine. 1998;23(5):537-542.

68. Rohlmann A, Bergmann G, Graichen F. Loads on an internal spinal fixation device during walking. J Biomech. 1996;30(1):41-47.

69. Rohlmann A, Gabel U, Graichen F, Bender A, Bergmann G. An instrumented implant for vertebral body replacement that measures loads in the anterior spinal column. Med Eng Phys. 2007;29(5):580-585.

70. Peterson J, Chlebek C, Ledet EH. Spinal fusion implant stiffness affects load sharing. Spine J. 2016;16(10):S260.

71. Rohlmann A, Graichen F, Bender A, Kayser R, Bergmann G. Loads on a telemeterized vertebral body replacement measured in three patients within the first postoperative month. Clin Biomech. 2008;23(2):147-158.

72. Rohlmann A, Pohl D, Bender A, et al. Activities of everyday life with high spinal loads. PLoS One. 2014;9(5):e98510.

73. Rohlmann A, Graichen F, Kayser R, Bender A, Bergmann G. Loads on a telemeterized vertebral body replacement measured in two patients. Spine. 2008;33(11):1170-1179.

74. Aebersold J, Hnat W, Voor M, et al. Development of a strain transferring sensor housing for a lumbar spinal fusion detection system. J Med Devices. 2007;1(2):159-164.

75. Lin J, Walsh K, Jackson D, et al. Development of capacitive pure bending strain sensor for wireless spinal fusion monitoring. Sens Actuators A. 2007;138(2):276-287.

76. Ferrara L, Gordon I, Coquillette M, et al. A preliminary biomechanical evaluation in a simulated spinal fusion model. J Neurosurg Spine. 2007;7(5):542-548

77. Rohlmann A, Bergmann G, Graichen F, Weber U. Comparison of loads on internal spinal fixation devices measured in vitro and in vivo. Med Eng Phys. 1997;19(6):539-546.

78. Zbinden D. Wireless Implantable Load Monitoring System for Scoliosis Surgery [master's thesis]. Alberta: University of Alberta; 2011.

79. Demetropoulos CK, Morgan CR, Sengupta DK, Herkowitz HN. Development of a 4-axis load cell used for lumbar interbody load measurements. Med Eng Phys. 2009;31(7):846-851.

80. Rohlmann A, Graichen F, Weber U, Bergmann G. Monitoring in vivo implant loads with a telemeterized internal spinal fixation device. Spine. 2000;25(23):2981-2986. 
81. Rohlmann A, Dreischarf M, Zander T, et al. Monitoring the load on a telemeterised vertebral body replacement for a period of up to 65 months. Eur Spine J. 2013;22(11):2575-2581.

82. Peterson J. Optimization of Spinal Instrumentation Stiffness and Its Effect on Interbody Fusion. Biomedical Engineering. Troy, NY: Rensselaer Polytechnic Institute; 2017.

83. Burny F, Zucman J, Bourgois R, et al. Utilisation des Jauges Extensometriques Pour la Mesure de la Consolidation des Fractures du Tibia Traitees par Enclouage Centromedullaire. Acta Orthop Belg. 1971;37:266-277.

84. Burny F, Donkerwolcke M, Bourgois R, Domb M, Saric O. Twenty years experience in fracture healing measurements with strain gauges. Orthopedics. 1984;7(12):1823-1826.

85. An K, Kasman R, Chao E. Theoretical analysis of fracture healing monitoring with external fixators. Eng Med. 1988;17(1):11-15.

86. Schneider E, Michel MC, Genge M, Zuber K, Ganz R, Perren SM. Loads acting in an intramedullary nail during fracture healing in the human femur. J Biomech. 2001;34(7):849-857.

87. Claes L, Cunningham J. Monitoring the mechanical properties of healing bone. Clin Orthop Relat Res. 2009;467(8):1964-1971.

88. Claes L, Grass R, Schmickal T, et al. Monitoring and healing analysis of 100 tibial shaft fractures. Langenbecks Arch Surg. 2002;387(3-4):146-152.

89. Bassey E, Littlewood J, Taylor S. Relations between compressive axial forces in an instrumented massive femoral implant, ground reaction forces, and integrated electromyographs from vastus lateralis during various 'osteogenic' exercises. J Biomech. 1997;30(3):213-223.

90. Burny F. Strain gague measurement of fracture healing. In: Brooker A, Cooney W, Chao E, editors. Principles of External Fixation. Baltimore: Williams and Wilkins; 1983:71-82.

91. Taylor SJ, Perry JS, Meswania JM, Donaldson N, Walker PS, Cannon SR. Telemetry of forces from proximal femoral replacements and relevance to fixation. J Biomech. 1997;30(3):225-234.

92. Taylor S, Walker P. Forces and moments telemetered from two distal femoral replacements during various activities. J Biomech. 2001;34(7):839-848.

93. Stoffel K, Klaue K, Perren S. Functional load of plates in fracture fixation in vivo and its correlate in bone healing. Injury. 2000;31(Suppl 2): SB37-SB50.
94. Sauer S, Marschner U, Adolphi B, et al. Passive wireless resonant Galfenol sensor for osteosynthesis plate bending measurement. IEEE Sens J. 2012;12(5):1226-1233.

95. Wilson DJ, Morgan RL, Hesselden KL, Dodd JR, Janna SW, Fagan MJ A single-channel telemetric intramedullary nail for in vivo measurement of fracture healing. J Orthop Trauma. 2009;23(10):702-709.

96. Panescu D. Emerging technologies [wireless communication systems for implantable medical devices]. IEEE Engineering in Medicine and Biology Magazine. 2008:96-101.

97. Silva NM, Santos PM, Ferreira JA, et al. Power management architecture for smart hip prostheses comprising multiple energy harvesting systems. Sens Actuators A Phys. 2013;202:183-192.

98. Ferrara L, Fleischman A, Togawa D, et al. An in vivo biocompatability assessment of MEMS materials for spinal fusion monitoring. Biomed Microdevices. 2003;5(4):297-302.

99. Alfaro F, Weiss L, Campbell P, et al. Design of a multi-axis implantable MEMS sensor for intraosseous bone stress monitoring. J Micromech Microeng. 2009;19:1-13.

100. Melik R, Perkgoz N, Unal E, et al. Bio-implantable passive on-chip RF-MEMS strain sensing resonators for orthopaedic applications. J Micromech Microeng. 2008;18:1-9.

101. Forchelet D, Simoncini M, Arami A, et al. Enclosed electronic system for force measurements in knee implants. Sensors (Basel). 2014;14(8):15009-15021.

102. Micolini C, Holness FB, Johnson JA, et al. Conductive polymer sensor arrays for smart orthopaedic implants. Nanosensors, Biosensors, Info-Tech Sensors and 3D Systems 2017: International Society for Optics and Photonics. 2017:101670D.

103. Wachs R, Ellstein D, Drazan J, et al. Elementary implantable force sensor for smart orthopaedic implants. Adv Biosens Bioelectron. 2013;2(4):12477.

104. Drazan J, Gunko A, Dion M, et al. Archimedean spiral pairs with no electrical connections as a passive wireless implantable sensor. J Biomed Technol Res. 2014;1:8.

105. Ledet E, Wachs R. Wireless implantable sensors with no electrical connections enable the next generation of smart orthopaedic implants. Bone Zone. 2012;6:82-84.
Innovation and Entrepreneurship in Health

\section{Publish your work in this journal}

Innovation and Entrepreneurship in Health is an international, peer reviewed, open access journal publishing original research, reports, reviews and commentaries on innovation and entrepreneurship in health. Special focus will be given to the theory, process, and practice of innovation and entrepreneurship by individuals and organizations

\section{Dovepress}

within the health care context globally. The manuscript management system is completely online and includes a very quick and fair peer review system, which is all easy to use. Visit http://www.dovepress.com/ testimonials.php to read real quotes from published authors. 